\title{
Acoustic emission across the magnetostructural transition of the giant magnetocaloric $\mathbf{G d}_{5} \mathbf{S i}_{2} \mathbf{G e}_{2}$
}

\author{
Francisco-José Pérez-Reche, ${ }^{1}$ Fèlix Casanova, ${ }^{2}$ Eduard Vives,${ }^{1}$ Lluís Mañosa,${ }^{1}$ Antoni Planes, ${ }^{1}$ Jordi Marcos, ${ }^{1}$ \\ Xavier Batlle, ${ }^{2}$ and Amílcar Labarta ${ }^{2}$ \\ ${ }^{1}$ Departament d'Estructura i Constituents de la Matèria, Facultat de Física, Universitat de Barcelona, Diagonal 647, \\ 08028 Barcelona, Catalonia, Spain \\ ${ }^{2}$ Departament de Física Fonamental, Facultat de Física, Universitat de Barcelona, Diagonal 647, 08028 Barcelona, Catalonia, Spain
}

(Received 16 September 2005; revised manuscript received 8 November 2005; published 20 January 2006)

\begin{abstract}
We report on the existence of acoustic emission during the paramagnetic-monoclinic $\leftrightarrow$ ferromagneticorthorhombic magnetostructural phase transition in the giant magnetocaloric $\mathrm{Gd}_{5} \mathrm{Si}_{2} \mathrm{Ge}_{2}$ compound. The transition kinetics have been analyzed from the detected acoustic signals. It is shown that this transition proceeds by avalanches between metastable states.
\end{abstract}

DOI: 10.1103/PhysRevB.73.014110

PACS number(s): 64.60.My, 64.70.Kb, 62.65.+k

\section{INTRODUCTION}

The interplay of magnetism and structure is at the origin of many of the technologically important properties of functional materials such as the giant magnetocaloric effect, magnetic shape memory, and giant magnetoresistance. ${ }^{1}$ Magnetocaloric effects associated with magnetic transitions have received a considerable amount of interest in recent years. ${ }^{2,3}$ $\mathrm{Gd}_{5}\left(\mathrm{Si}_{x} \mathrm{Ge}_{1-x}\right)_{4}$ intermetallics are prominent among the materials exhibiting such effect and have received a great deal of attention. ${ }^{4,5}$ In these compounds, the giant magnetocaloric effect is due to the occurrence of a magnetic phase transition which also involves a crystallographic structural change. ${ }^{6,7}$ This transition is first order, reversible, and can be induced either by changing the temperature, ${ }^{6,7}$ the pressure, ${ }^{8,9}$ or by application of a magnetic field. ${ }^{7,10}$ In the range $0.24 \leqslant x \leqslant 0.5$, the transition goes from a paramagnetic monoclinic phase towards a ferromagnetic orthorhombic phase on cooling. ${ }^{11}$

A structural change in a material usually produces changes in the internal strain field, which give rise to elastic waves in the ultrasonic range propagating within the material. These elastic waves are known as acoustic emission (AE) and they convey information on the dynamics of the mechanism that has generated them. ${ }^{12}$ Prototypical solidsolid phase transitions with associated $\mathrm{AE}$ are martensitic transitions, for which the analysis of the $\mathrm{AE}$ has provided valuable information on the mechanisms and kinetics of the transition. ${ }^{12-16}$

The mechanisms of the crystallographic change in $\mathrm{Gd}_{5}\left(\mathrm{Si}_{x} \mathrm{Ge}_{1-x}\right)_{4}$ involve shearing of planes perpendicular to the long $b$ axis. ${ }^{6,17}$ Since this mechanism shares some similarities with martensitic transitions, it has been termed "martensiticlike." Hence, it is expected that such a structural change can generate $\mathrm{AE}$ in these kinds of materials. In the present paper we show the existence of $\mathrm{AE}$ during the magnetostructural transition of $\mathrm{Gd}_{5} \mathrm{Si}_{2} \mathrm{Ge}_{2}$ and we analyze the kinetics of the transition from the detected AE. Results are discussed in comparison with the well-established $\mathrm{AE}$ results in thermoelastic martensitic transitions.

\section{EXPERIMENTAL DETAILS}

A $\mathrm{Gd}_{5} \mathrm{Si}_{2} \mathrm{Ge}_{2}$ sample was synthesized by arc melting the pure elements (commercial 99.9 wt \% Gd, 99.9999 wt \% Si and 99.999 wt \% Ge) in the appropriate stoichiometry under a high-purity argon atmosphere. The sample was placed in a water-cooled copper crucible and was melted several times to ensure good homogeneity. From the as-prepared buttons, the sample was cut with two parallel faces and it was heat treated for homogenization for up to $9 \mathrm{~h}$ at $950{ }^{\circ} \mathrm{C}$ under $10^{-5}$ mbar, inside a quartz tube in an electrical resistance furnace. After annealing, the quartz tube was quickly taken out of the furnace and was left to cool to room temperature. The crystallographic structure of the sample was studied by room-temperature $\mathrm{x}$-ray diffraction. The material displayed the expected monoclinic structure $\left(P 112_{1} / a\right)$ with unit cell parameters $a=7.577(1), \quad b=14.790(3), \quad c=7.779(1)$, $\gamma=93.09(1)$, in agreement with Ref. 18. Some amounts of a secondary orthorhombic phase (Pnma) were also present in the sample.

$\mathrm{AE}$ signals were detected by a resonant piezoelectric transducer acoustically coupled to the surface of the sample. The transition was thermally induced using the experimental setup described in Ref. 15. The relative oscillations of the sample temperature were less than $0.01 \%$. The amplified signal (gain $62 \mathrm{~dB}$ ) was simultaneously processed by two different methods. On the one hand, bursts with amplitudes exceeding a fixed threshold were stored using a digitizing oscilloscope, which is capable of recording $10^{4} \mathrm{AE}$ pulses (1000 points per signal at $1 \mathrm{MHz}$ ) during the magnetostructural transition. On the other hand, the signal was input into a ring-down frequency meter which renders the count rate $\dot{N}=d N / d t$ (number of signals recorded during $1 \mathrm{~s}$ ).

\section{RESULTS AND DISCUSSION}

Figure 1 shows an example of the reduced counting rate $(r=d N / d T=\dot{N} / \dot{T})$ recorded as a function of temperature. A clear increase in AE activity is observed for the $280-260 \mathrm{~K}$ temperature range on cooling and $265-290 \mathrm{~K}$ on heating. These ranges coincide with those where the magnetostructural transition takes place, as determined from calorimetric measurements done on the same sample, and thus confirm that there is $\mathrm{AE}$ generated during the transition. Acoustic activity across the monoclinic to orthorhombic (cooling) 
transition is higher than across the reverse transition. Differences in the kinetics of the transition between cooling and heating were also reported in $\mathrm{Gd}_{5} \mathrm{Si}_{1.9} \mathrm{Ge}_{2.05}$ from voltage generation measurements. ${ }^{19}$ It is worth noting that the recorded $\mathrm{AE}$ is very weak during both heating and cooling runs, as compared, for instance, to the activity recorded during thermoelastic martensitic transitions for which the overall number of recorded counts typically exceeds the $\mathrm{AE}$ recorded here by more than three orders of magnitude. ${ }^{20}$

The reproducibility of the AE pattern during the magnetostructural transition was studied. To this end, we performed up to $\sim 30$ complete thermal cycles. A quantitative evaluation of the similarity in the AE pattern is achieved by computing the correlation function $\rho_{n, n+1}$ between the reduced AE activity of two consecutive cycles defined as ${ }^{21}$

$$
\rho_{n, n+1}=\frac{\int_{0}^{1} r_{n} r_{n+1} d \tau-\int_{0}^{1} r_{n} d \tau \int_{0}^{1} r_{n+1} d \tau^{\prime}}{\sqrt{\left[\int_{0}^{1} r_{n}^{2} d \tau-\left(\int_{0}^{1} r_{n} d \tau\right)^{2}\right]\left[\int_{0}^{1} r_{n+1}^{2} d \tau-\left(\int_{0}^{1} r_{n+1} d \tau\right)^{2}\right]}},
$$

where $\tau \equiv\left(T-T_{i}\right) /\left(T_{f}-T_{i}\right), T_{i}$ is a temperature above (below) the starting transition temperature on cooling (heating), and $T_{f}$ is a temperature below (above) the finishing transition temperature on cooling (heating). The value of $\rho_{n, n+1}$ quantifies how much the reduced acoustic activity as a function of temperature in the $n$th cycle resembles that of the $(n+1)$ th. When $r_{n}$ is very similar to $r_{n+1}, \rho_{n, n+1}$ is close to unity.

The correlation function $\rho_{n, n+1}$ versus the cycle number $n$ is presented in Fig. 2 for cooling and heating runs. In both cases, a clear increase in correlation is observed with cycling, which indicates that the transition becomes progressively more reproducible. Such an increase is very fast for the reverse transition (heating). It occurs for the first 7-8 cycles, after which it saturates. In contrast, the increase is smoother on cooling.

Acoustic activity during the magnetostructural transition

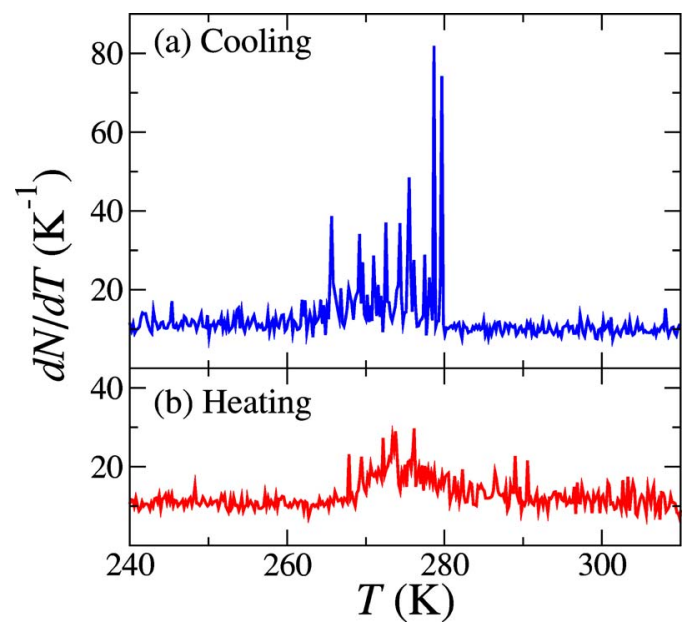

FIG. 1. (Color online) Reduced acoustic activity recorded during the 13th cycle on (a) cooling and (b) heating. corresponds to individual AE signals (bursts) which is a signature of the jerky character of the transition. We also performed a statistical analysis of the individual signals recorded during cooling. These runs are carried out once the system has been cycled a sufficient number of times for the transition to be reproducible. The low number of signals recorded during heating prevented us from performing a reliable statistical analysis of the reverse transition. Figure 3 shows the $\mathrm{AE}$ amplitude distribution $p(A)$ of the signals recorded at cooling rates $(\dot{T}) 1 \mathrm{~K} / \mathrm{min}$ and $6 \mathrm{~K} / \mathrm{min}$. In addition, to improve statistics, we used all the signals recorded during 40 and 170 cycles for $\dot{T}=1 \mathrm{~K} / \mathrm{min}$ and $\dot{T}=6 \mathrm{~K} / \mathrm{min}$, respectively. The data exhibit an apparent power-law behavior which evidences the absence of a characteristic scale. A more quantitative analysis can be performed by fitting the following probability density with two free parameters, the exponent $\alpha$ and the exponential correction $\lambda$,

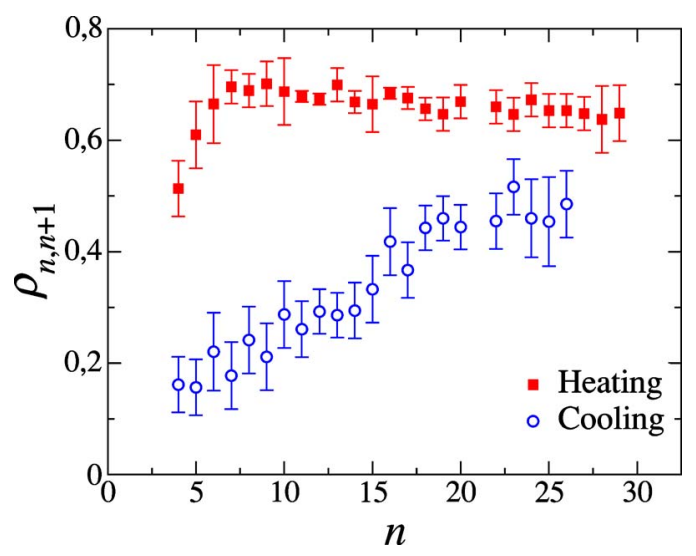

FIG. 2. (Color online) Correlation function between reduced acoustic activity of consecutive cycles. Data corresponding to cooling and heating runs are represented by different symbols as indicated by the legend. 


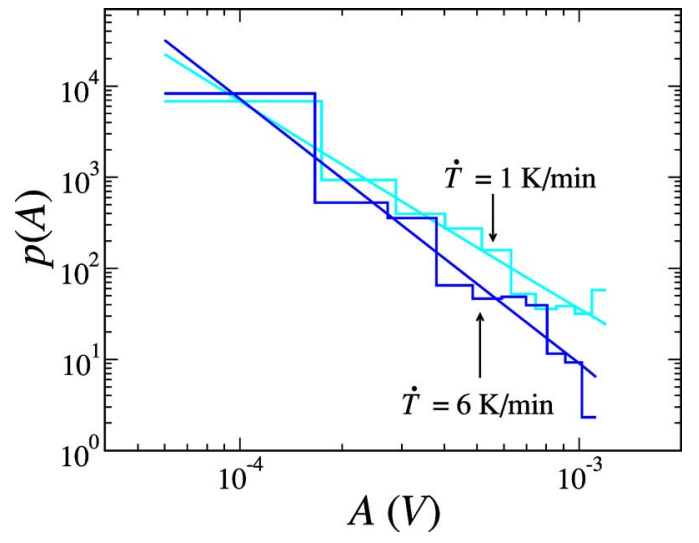

FIG. 3. (Color online) Normalized histograms corresponding to the distribution of amplitudes $p(A)$ of $\mathrm{AE}$ signals recorded during cooling runs at two different cooling rates, $\dot{T}=1 \mathrm{~K} / \mathrm{min}$ and $\dot{T}=6 \mathrm{~K} / \mathrm{min}$. Straight lines indicate the fits to the histograms.

$$
p(A)=\frac{e^{-\lambda A} A^{-\alpha}}{\int_{A_{\min }}^{A_{\max }} e^{-\lambda A} A^{-\alpha} d A},
$$

where $A$ is the amplitude of the signals. The normalization factor in the denominator corresponds to the integral of the proposed distribution from the minimum to the maximum of the acquired amplitudes: $A_{\min }=6 \times 10^{-5} \mathrm{~V}$ and $A_{\max }=1.123 \times 10^{-3} \mathrm{~V}$. The estimation of the parameters $\alpha$ and $\lambda$ is done by using the maximum likelihood method. ${ }^{21,22}$ This method is much more reliable than the standard least squares method since it is not based on the computation of the histograms which usually depend on the binning choice, and in addition it forces the fitted probability density to be normalized.

The obtained values are $\alpha=2.33 \pm 0.03$ and $\lambda$ $=-130 \pm 120 \mathrm{~V}^{-1}$ for $\dot{T}=1 \mathrm{~K} / \mathrm{min}$ and $\alpha=2.90 \pm 0.05$ and $\lambda=0 \pm 246 \mathrm{~V}^{-1}$ for $\dot{T}=6 \mathrm{~K} / \mathrm{min}$. The value of $\lambda$ is compatible with $\lambda=0$ in both cases, which indicates that the distribution is well described by a power-law.

The magnetostructural transition in $\mathrm{Gd}_{5} \mathrm{Si}_{2} \mathrm{Ge}_{2}$ has been compared by several authors with martensitic transitions which also occur via a shear mechanism. As a particular example, the shear distortion observed in thermoelastic martensitic transitions in $\mathrm{Cu}$-based alloys is about $0.12 .{ }^{23}$ Such a shear distortion is larger than that involved in the magnetostructural transition in $\mathrm{Gd}_{5} \mathrm{Si}_{2} \mathrm{Ge}_{2}$, which is $\sim 0.03 .{ }^{17}$ Such a difference may be considered as a possible reason for the lower acoustic activity detected across the magnetostructural transition.

There is a fundamental difference between a martensitic transition and the magnetostructural transition in $\mathrm{Gd}_{5} \mathrm{Si}_{2} \mathrm{Ge}_{2}$. From a purely crystallographic point of view the martensitic transition occurs, on cooling, from a high-symmetry phase to a low-symmetry phase. In contrast, the transition in $\mathrm{Gd}_{5} \mathrm{Si}_{2} \mathrm{Ge}_{2}$ occurs from a low-symmetry phase (monoclinic) to a high-symmetry phase (orthorhombic). Such a symmetry change does not fit the common framework of phase transi- tions since it is expected that the high-symmetry phase corresponds to the high-temperature phase. Nevertheless, the transition in $\mathrm{Gd}_{5} \mathrm{Si}_{2} \mathrm{Ge}_{2}$ also involves a symmetry change associated with magnetic degrees of freedom from a paramagnetic phase (high symmetry) to a ferromagnetic phase (low symmetry). Hence, symmetry arguments indicate that the main driving force for the magnetostructural phase transition arises from the magnetic degrees of freedom. It is worth noting that first principle calculations ${ }^{24,25}$ have shown that exchange coupling is higher in the orthorhombic phase, which stabilizes that phase at low temperatures. Also, for $\mathrm{Gd}_{5} \mathrm{Ge}_{4}$, it was shown ${ }^{26}$ that magnetic degrees of freedom play a primary role in driving the magnetostructural transition in that compound.

From the above arguments concerning crystallographic changes, the properties of the forward martensitic transition must be compared with those of the reverse magnetostructural transition, and vice versa. Two independent experimental findings are consistent with this point of view. The first is the fact that the amount of AE across the forward magnetostructural transition is greater than across the reverse transition, while, typically for martensitic transitions, AE is greater during the reverse transition. ${ }^{20}$ The second finding is concerned with nucleation. According to TEM experiments, ${ }^{17}$ no evidence of nucleation of the orthorhombic phase was observed on cooling $\mathrm{Gd}_{5} \mathrm{Si}_{2} \mathrm{Ge}_{2}$. This observation is consistent with the fact that there is no nucleation for the reverse (lowsymmetry to high-symmetry) martensitic transition.

Our results show that acoustic activity evolves towards a more reproducible pattern with thermal cycling through the magnetostructural transition. Actually, some dependence on thermal cycling has been previously reported in resistance, ${ }^{27,28}$ thermopower, ${ }^{29}$ and calorimetric measurements ${ }^{30}$ in $\mathrm{Gd}_{5}\left(\mathrm{Si}_{x} \mathrm{Ge}_{1-x}\right)_{4}$ alloys. Evolution with thermal cycling is frequently observed in first-order structural phase transitions as, for instance, martensitic transformations. ${ }^{21}$ Such evolution has been interpreted as a learning process in which the system seeks an optimal path for the transition that tends to reduce the dissipated energy and therefore the width of the hysteresis loop. During this learning process the characteristics of the transition evolve towards a reproducible pattern and the entropy change decreases. In $\mathrm{Gd}_{5}\left(\mathrm{Si}_{x} \mathrm{Ge}_{1-x}\right)_{4}$ such evolution has been associated with the creation of microcracks during cycling. ${ }^{27}$ This interpretation is, in fact, analogous to the creation of defects (mainly dislocations) during martensitic transition. From these arguments it seems reasonable to associate the evolution in $\mathrm{Gd}_{5} \mathrm{Si}_{2} \mathrm{Ge}_{2}$ to the structural degrees of freedom involved in the transition.

AE is a very sensitive technique to study the kinetics of a structural transition. Present results show that the temperature-driven magnetostructural transition in $\mathrm{Gd}_{5} \mathrm{Si}_{2} \mathrm{Ge}_{2}$ proceeds by multiple steps (avalanches) joining a series of metastable states, until the system is fully transformed. This behavior has been encountered in a wide variety of driven processes in different systems (reversal of the magnetization both in standard metamagnetic transitions ${ }^{31}$ and magnetostructural transitions, ${ }^{32}$ martensitic transition, ${ }^{14,33}$ emergence of vortices in superconductors, ${ }^{34}$ vapor condensation in porous media, ${ }^{35}$ etc.). While it is not 


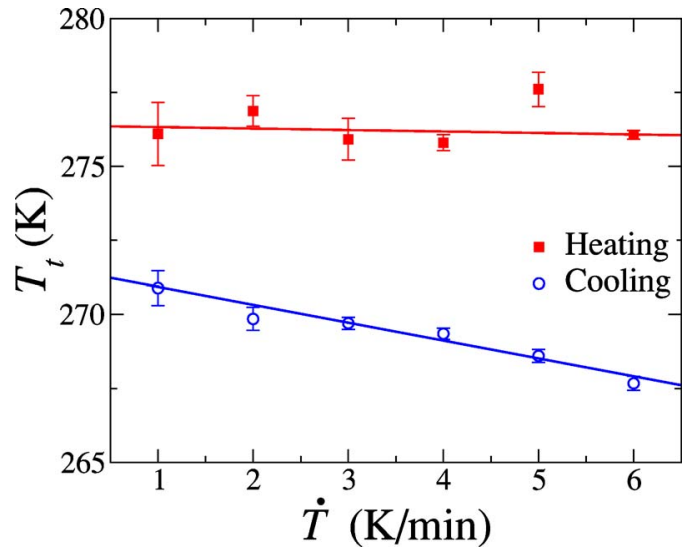

FIG. 4. (Color online) Average values of the transition temperatures according to Eq. (3) for cooling and heating runs as a function of $\dot{T}$. Data corresponding to cooling and heating runs are indicated by different symbols according to the legend. Continuous lines are linear fits to the data.

obvious that these systems should show similar properties, they share a common characteristic of being spatially extended, with a complex free energy landscape. Quenched-in disorder is at the origin of such a complex landscape. For all these systems, there is a lack of characteristic duration and size scales of these avalanches, as reflected by power-law distributions. As illustrated in Fig. 3, the amplitude of AE signals recorded here follows a power-law distribution, thus showing that the magnetostructural transition in $\mathrm{Gd}_{5} \mathrm{Si}_{2} \mathrm{Ge}_{2}$ belongs to such a general class of systems.

The kinetics of these avalanche-mediated, first-order phase transitions has been acknowledged to be athermal (i.e., thermal fluctuations are not dominant when driving the system from one phase to another). The effect of thermal fluctuations (athermal degree) and also the effect of driving rate on the power-law exponents has been explained in terms of the different time scales involved in driving the transition. ${ }^{15,33}$ Comparison of the amplitude distributions obtained for $\dot{T}=1 \mathrm{~K} / \mathrm{min}$ and $\dot{T}=6 \mathrm{~K} / \mathrm{min}$ shows that the power-law exponent for cooling runs increases for increasing temperature rate. This is the behavior expected for those transitions for which the characteristic time of thermal fluctuations is not very different from the characteristic time associated with the change of the external field (temperature in the present case). In order to provide further support to this statement, we measured the transition temperature as a function of the temperature rate. For those transitions in which thermal fluctuation cannot be neglected, it is expected that the transition temperature decreases on increasing the temperature rate. In contrast, no dependence is expected for athermal transitions. In Fig. 4 we present the average transi- tion temperature $T_{t}$ for forward and reverse magnetostructural transitions as a function of the temperature rate. Each data point in the figure corresponds to an average over 10 cycles for each driving rate. Due to the weakness of the detected $\mathrm{AE}$, determination of the transition temperature is not straightforward and we have estimated it as a weighted average of $T$ with the reduced counting rate $r$ as the weighting factor,

$$
T_{t}=\frac{\int_{T_{i}}^{T_{f}} \operatorname{Tr}(T) d T}{\int_{T_{i}}^{T_{f}} r(T) d T} .
$$

As shown in Fig. 4, a clear decrease of the transition temperature for a monoclinic to orthorhombic transition is observed while the orthorhombic to monoclinic transition shows no significant dependence. The fact that thermal fluctuations are more relevant for the forward transition than for the reverse one is also consistent with the results presented in Fig. 2. Athermal transitions are expected to be much more reproducible than those which are affected by thermal fluctuations.

Our results are consistent with the finding of thermally activated effects observed in the paramagnetic to ferromagnetic field-induced transition in $\mathrm{Gd}_{5} \mathrm{Si}_{2} \mathrm{Ge}_{2}{ }^{36}$ In this case the application of a magnetic field induces a sudden increase in magnetization followed by a smooth thermally activated relaxation towards a saturation value. Since our experiments indicate a more athermal character on heating, we suggest that the relaxation process observed after a demagnetizing process (ferromagnetic-monoclinic $\rightarrow$ paramagneticorthorhombic) should be slower than that observed when the magnetic field increases.

\section{CONCLUSION}

We have demonstrated the existence of acoustic emission across the magnetostructural transition in $\mathrm{Gd}_{5} \mathrm{Si}_{2} \mathrm{Ge}_{2}$. The burstlike character of the recorded $\mathrm{AE}$ reflects the jerky character of the transition. This evidences the fact that the magnetostructural transition in giant magnetocaloric materials proceeds by avalanches between metastable states. Moreover, there is no characteristic scale for the size of these avalanches. The experimental results also show that thermal fluctuations do not play a relevant role (athermal character) for the orthorhombic $\rightarrow$ monoclinic transition.

\section{ACKNOWLEDGMENTS}

This work has received financial support from CICyT (Spain), Projects No. MAT2004-1291 and No. MAT200301124 and DURSI (Catalonia), Project No. 2005SGR00969. 
*Electronic address: 1luis@ecm.ub.es

${ }^{1}$ A. Planes, L. Mañosa, and A. Saxena, Interplay of Magnetism and Structure in Functional Materials edited by A. Planes, L. Mañosa, and A. Saxena, Materials Science Series Vol. 79 (Springer-Verlag, Berlin, 2005).

${ }^{2}$ K. A. Gschneidner, Jr., V. K. Pecharsky, and A. O. Tsokol, Rep. Prog. Phys. 68, 1479 (2005).

${ }^{3}$ O. Tegus, E. Brück, L. Zhang, K. H. J. Buschow, and F. R. de Boer, Physica B 319, 174 (2002).

${ }^{4}$ V. K. Pecharsky and K. A. Gschneidner Jr., Phys. Rev. Lett. 78, 4494 (1997).

${ }^{5}$ V. Provenzano, A. J. Shapiro, and R. D. Schull, Nature 429, 853 (2004).

${ }^{6}$ W. Choe, V. K. Pecharsky, A. O. Pecharsky, K. A. Gschneidner, Jr., V. G. Young, Jr., and G. J. Miller, Phys. Rev. Lett. 84, 4617 (2000).

${ }^{7}$ L. Morellon, P. A. Algarabel, M. R. Ibarra, J. Blasco, B. GarcíaLanda, Z. Arnold, and F. Albertini, Phys. Rev. B 58, R14721 (1998).

${ }^{8}$ Y. Mudryk, Y. Lee, T. Vogt, K. A. Gschneidner, Jr., and V. K. Pecharsky, Phys. Rev. B 71, 174104 (2005).

${ }^{9}$ L. Morellon, Z. Arnold, P. A. Algarabel, C. Magen, M. R. Ibarra, and Y. Skorokhod, J. Phys.: Condens. Matter 16, 1623 (2004).

${ }^{10}$ F. Casanova, A. Labarta, X. Batlle, J. Marcos, L. Mañosa, A. Planes, and S. de Brion, Phys. Rev. B 69, 104416 (2004).

${ }^{11}$ L. Morellon, J. Blasco, P. A. Algarabel, and M. R. Ibarra, Phys. Rev. B 62, 1022 (2000).

${ }^{12}$ L. Mañosa, A. Planes, D. Rouby, M. Morin, P. Fleischman, and J. L. Macqueron, Appl. Phys. Lett. 54, 2574 (1989).

${ }^{13}$ Z. Yu and P. C. Clapp, J. Appl. Phys. 62, 2212 (1987).

${ }^{14}$ E. Vives, J. Ortín, L. Mañosa, I. Ràfols, R. Pérez-Magrané, and A. Planes, Phys. Rev. Lett. 72, 1694 (1994).

${ }^{15}$ F. J. Pérez-Reche, E. Vives, L. Mañosa, and A. Planes, Phys. Rev. Lett. 87, 195701 (2001).

${ }^{16}$ L. Mañosa, A. Planes, D. Rouby, and J. L. Macqueron, Acta Metall. Mater. 38, 1635 (1990).

${ }^{17}$ J. S. Meyers, S. Chumbley, F. Laabs, and A. O. Pecharsky, Acta Mater. 51, 61 (2003).
${ }^{18}$ V. Pecharsky and K. A. Gschneidner, Jr., J. Alloys Compd. 260, 98 (1997).

${ }^{19}$ E. M. Levin, V. K. Pecharsky, and K. A. Gschneidner, Jr., Phys. Rev. B 63, 174110 (2001).

${ }^{20}$ L. Mañosa, A. Planes, and E. Cesari, J. Phys. D 22, 977 (1989).

${ }^{21}$ F. J. Pérez-Reche, M. Stipcich, E. Vives, L. Mañosa, A. Planes, and M. Morin, Phys. Rev. B 69, 064101 (2004).

${ }^{22}$ M. L. Goldstein, S. A. Morris, and G. G. Yen, Eur. Phys. J. B 41, 255 (2004)

${ }^{23}$ G. Guénin and P. F. Gobin, J. Phys. (Paris) 43, C4 (1982).

${ }^{24}$ G. Samolyuk and V. Antropov, J. Appl. Phys. 97, 10A310 (2005).

${ }^{25}$ V. K. Pecharsky, K. A. Gschneidner, Jr., G. D. Samolyuk, V. P. Antropov, A. O. Pecharsky, and K. A. Gschneidner, Jr., J. Solid State Chem. 171, 57 (2003).

${ }^{26}$ V. K. Pecharsky, A. P. Holm, K. A. Gschneidner, Jr., and R. Rink, Phys. Rev. Lett. 91, 197204 (2003).

${ }^{27}$ E. M. Levin, A. O. Pecharsky, V. K. Pecharsky, and K. A. Gschneidner, Jr., Phys. Rev. B 63, 064426 (2001).

${ }^{28}$ J. B. Sousa, M. E. Braga, F. C. Correia, F. Carpinteiro, L. Morellon, P. A. Algarabel, and M. R. Ibarra, Phys. Rev. B 67, 134416 (2003).

${ }^{29}$ J. B. Sousa, M. E. Braga, F. C. Correira, F. Carpinteiro, L. Morellon, P. A. Algarabel, and M. R. Ibarra, J. Appl. Phys. 91, 4457 (2002).

${ }^{30}$ F. Casanova, A. Labarta, X. Batlle, F. J. Pérez-Reche, E. Vives, L. Mañosa, and A. Planes, Appl. Phys. Lett. 86, 262504 (2005).

${ }^{31}$ G. Durin and S. Zapperi, in The Science of Hysteresis, edited by I. Mayergoyz and G. Bertotti (Academic Press, 2006).

${ }^{32}$ V. Hardy, S. Majumdar, M. R. Lees, D. McK. Paul, C. Yaicle, and M. Hervieu, Phys. Rev. B 70, 104423 (2004).

${ }^{33}$ F. J. Pérez-Reche, B. Tadić, L. Mañosa, A. Planes, and E. Vives, Phys. Rev. Lett. 93, 195701 (2004).

${ }^{34}$ E. Altshuler and T. H. Johansen, Rev. Mod. Phys. 76, 471 (2004), and refs. therein.

${ }^{35}$ M. P. Lilly, P. T. Finley, and R. B. Hallock, Phys. Rev. Lett. 71, 4186 (1993).

${ }^{36}$ J. Leib, J. E. Snyder, T. A. Lograsso, D. Schlagel, and D. C. Jiles, J. Appl. Phys. 95, 6915 (2004). 